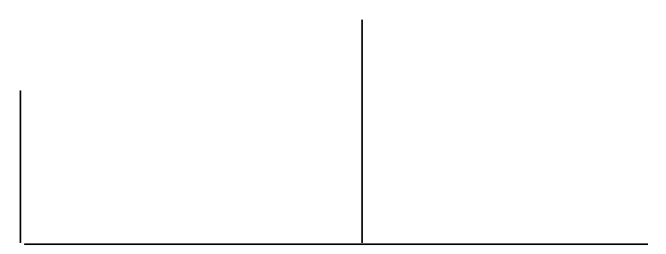

Rev. Latinoam. Psicopat. Fund., VI, 1, 100-108

\title{
Uma provável caracterização do profissional de Saúde Mental que atende dependentes de drogas no Vale do Paraíba*
}

\author{
Manuel Morgado Rezende
}

\begin{abstract}
Esta pesquisa foi realizada com profissionais de Saúde Mental, de diversas formações de nível universitário que atendem drogadependentes nas cidades de Taubaté e São José dos Campos, localizadas no Vale do Paraíba, Estado de São Paulo. Investigaramse características relativas à formação, atuação e alguns dos pontos de vista e observações destes profissionais sobre a experiência de atendimento a pacientes que apresentam transtornos relacionados a substâncias. Os diálogos com os entrevistados indicaram que há insatisfação com os resultados obtidos e com as condições de trabalho. Praticamente não há cursos de reciclagem, controle de eficácia, ou follow-up. A improvisação e o uso de técnicas ecléticas predominam sobre as intervenções definidas e avaliadas sistematicamente. A experiência assistencial é dominante em relação à de pesquisa, ou de atualização da literatura científica especializada.
\end{abstract}

Palavras-chave: Profissionais de saúde mental, experiência, atendimento, dependentes de drogas

* Este texto, parcialmente alterado, faz parte da tese de doutorado Tratamento de dependentes de drogas - diálogos com profissionais da área de Saúde Mental, defendida em 1999 na Faculdade de Ciências Médicas da Unicamp, com orientação do Prof. Dr. Isac Germano Karniol e bolsa do CNPq e da Unitau. 


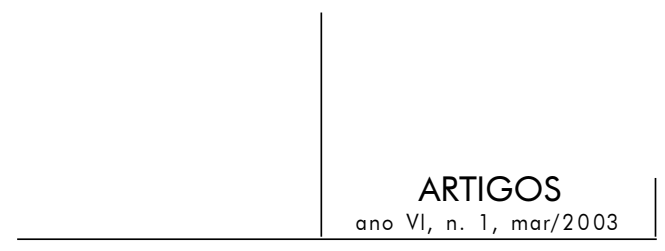

Introdução

Os sujeitos da pesquisa são profissionais da área de Saúde Mental que atendem usuários de drogas. A amostra foi composta por profissionais das cidades de Taubaté e São José dos Campos, localizadas no Vale do Paraíba, Estado de São Paulo.

Os entrevistados foram selecionados a partir de um conjunto de critérios que exigia, como condição mínima para inclusão na pesquisa, que o profissional tivesse alguma experiência no atendimento a consumidores de drogas. A maioria dos entrevistados são considerados, pelos colegas e (ou) chefes (no serviço público), os profissionais com melhor formação ou com mais disponibilidade para tratarem de transtornos relacionados a substâncias.

Optamos pela inclusão, na amostra estudada, de profissionais das cidades de Taubaté e São José dos Campos, por serem estas as maiores e mais populosas cidades do Vale do Paraíba. Ambas possuem universidades que formam profissionais nas carreiras pesquisadas: a Unitau (Universidade de Taubaté) tem cursos de graduação em medicina, enfermagem, serviço social e psicologia; e a Univap (Universidade do Vale do Paraíba) possui graduação em serviço social. Outros aspectos considerados foram o da facilidade geográfica de acesso aos profissionais, bem como o da tendência dos estudos em saúde mental definirem amostras de população regionalizadas.

Embora a presente pesquisa tenha como objetivo caracterizar e discutir aspectos ligados à formação e à experiência do profissional que atende dependentes de drogas, não podemos deixar de destacar a importância do contexto institucional em que as intervenções são efetuadas.

No presente estudo, utilizamos a entrevista como meio de coleta de dados. No contexto da entrevista, o sujeito entrevistado não se limita a um papel passivo, como num interrogatório ou num exame técnico, os quais enfatizam a neutralidade. Ao contrário, coloca-se, desde o início, como objeto ativo, organizador de um modo próprio de comunicação com o entrevistador (Bergeret, 1983). O fato deste último interferir com a sua presença, postura, atitudes e comportamentos, isentos ou não, faz dele um participante do diálogo e, portanto, ele é fator de interação objetiva e subjetiva 


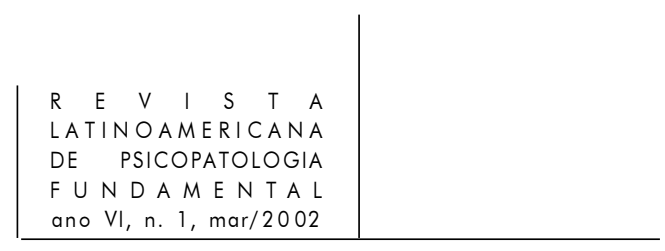

no campo da observação. Sendo assim, não podemos desconsiderar a condição do entrevistador como parte indissociável das observações dos dados e dos seus diversos níveis de interpretação e elaboração dos mesmos (Rezende, 1999).

Após a entrevista, aplicamos um questionário visando identificar a formação do profissional e sua atividade no atendimento a usuários de drogas. A discussão dos resultados foi baseada na análise de conteúdo (Bardin, 1977).

\section{Caracterização}

\section{Sujeitos: 22}

Idade (em anos): 40,3

Sexo: $59,1 \%$ do sexo masculino e $40,9 \%$ do sexo feminino.

Estado civil: $50 \%$ casados; $27,3 \%$ divorciados; $4,5 \%$ separados e $18,2 \%$ solteiros.

Curso de graduação: $18,2 \%$ enfermagem; $27,3 \%$ medicina; $27,3 \%$ psicologia; $18,2 \%$ serviço social; $9,1 \%$ terapia ocupacional.

Titulação acadêmica: 18,25\% com mestrado; 9,1\% cursaram créditos em mestrado; 72,3\% com curso de especialização em diversas áreas. Não há relato de curso de especialização em dependência de drogas. Os médicos e os psicólogos têm maior titulação acadêmica.

Tempo de trabalho, em anos, com usuários de drogas: tempo médio de 13,1; menor tempo $=2$; maior tempo $=33 ; \mathrm{n}^{\mathrm{o}}=22$ entrevistados. Os médicos e os assistentes sociais apresentam as maiores médias de tempo de trabalho, seguidos dos psicólogos, terapeutas ocupacionais e enfermeiros.

Tempo médio, em anos, para iniciar na área após o término da graduação: enfermagem 7,3; medicina 5,6; psicologia 2,8; serviço social 5,3; terapia ocupacional 0,5 .

Número médio de pacientes atendidos por semana: 17,2 pacientes. Mínimo 1; máximo 80.

Número médio de famílias atendidas por semana: 9,5 famílias. Mínimo 1; máximo 30.

Publicação de artigos: 1

Divulgação de informações para a população: 11 proferiram palestras em escolas; 8 em empresas.

Apresentação de trabalhos em congressos ou simpósios científicos na área: 1.

Presença em congressos ou simpósios da área: 12 entrevistados já compareceram. Assim distribuídos: 5 psicólogos, 4 médicos, 2 assistentes sociais, 1 enfermeiro. 


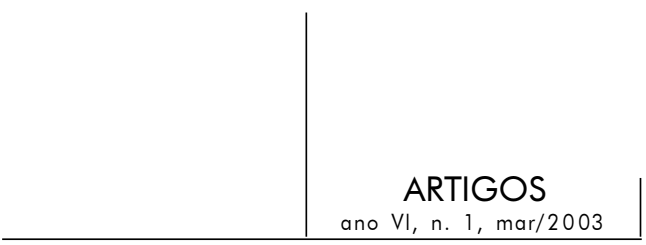

Motivações para atender usuários de drogas: $63 \%$ surge da demanda, sem motivação específica; 18,2\% não gostam de atender essa população; $18,2 \%$ pelo desafio e importância social "do problema"; $13,6 \%$ tem familiar dependente de drogas.

Objetivos específicos do tratamento: $54,5 \%$ abstinência/recuperação; $22,7 \%$ melhora a qualidade de vida; $9,0 \%$ depende das condições do paciente; $4,5 \%$ solucionar os problemas dos pacientes; $4,5 \%$ promover desenvolvimento mental; $4,5 \%$ oferecer o método psicoterápico.

Métodos e técnicas utilizadas: 4,5\% farmacoterapia; 4,5\% psicanálise; $4,5 \%$ psicodrama; $4,5 \%$ doze passos; $4,5 \%$ terapia centrada no cliente; $77,5 \%$ misturam diversas abordagens: internação, farmacoterapia, psicanálise, psicoterapia individual e de grupo, orientação familiar, aconselhamento, dinâmica de grupo, informações, doze passos entre outras. O ecletismo no uso de técnicas prevalece para se atingir o objetivo genérico de recuperação ou abstinência.

Resultados obtidos: $72,7 \%$ a recuperação foi de poucos; $18,2 \%$ melhoras em geral; 9,0\% depende das condições do paciente.

Gratificação pessoal e profissional: 40,9 frustrante; 36,3\% o mínimo que se consegue é gratificante; 9,0\% acrescenta experiência profissional; $9,0 \%$ nada específico; $4,5 \%$ deveria ganhar mais; $4,5 \%$ resgatar a minha juventude.

Dificuldades encontradas pelos profissionais: $50 \%$ adesão ao tratamento; $31 \%$ recaídas; $13,6 \%$ compulsão; $9 \%$ agressividade dos pacientes; $9 \%$ boicote familiar; $27,2 \%$ baixos salários; $9 \%$ medo de traficantes; $40,9 \%$ pressão social por resultados/falta de condições de trabalho; $9 \%$ compreensão teórica; $13,6 \%$ dificuldade de lidar com os pacientes; $12,7 \%$ falta de reciclagem/cursos/supervisão.

Dificuldades de colegas e outras genéricas: 13,6 trabalhar em equipe multiprofissional; 13,6\% falta de capacitação/supervisão; $9 \%$ excesso de trabalho/ estamos doentes; $4,5 \%$ estresse dos profissionais; $9 \%$ falta de conhecimentos e habilidades para lidar com usuários de drogas.

Fontes de informação: 27,2\% livros/jornais/televisão/revistas; 13,6\% artigos de especialistas conhecidos/supervisão; 27,2\% nenhuma leitura específica; $18,2 \%$ A.A ou N.A; $18,2 \%$ troca de experiências com colegas; $13,6 \%$ publicações do CEBRID; 9\% livros de Saúde Mental.

Registro da experiência: $50 \%$ apenas prontuário; 45,5\% levantamento de dados sócio-demográficos; $4,5 \%$ registro e estudo do material clínico.

Compreensão do uso, abuso, dependência de drogas: predomínio de pontos de vista ecléticos $(95,5 \%)$, apenas um entrevistado utiliza exclusivamente o ponto de vista biológico (4\%). O mais frequiente é a associação do modelo psicossocial com o biológico. As explicações do fenômeno agregam fatores biológicos (neurotransmissores, genéticos), de personalidade (baixa auto-estima, depressão, psicoses, psicopatias), familiares (rigidez, permissividade, ausência paterna, rejeição, falta de diálogo) e socioculturais (preconceitos, hipocrisia, omissão das 


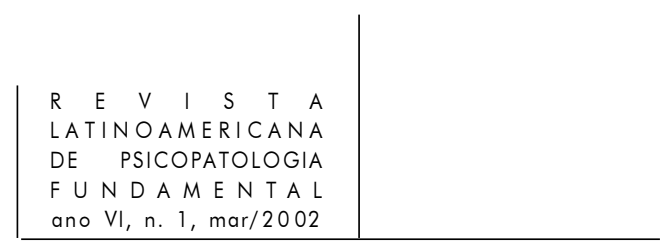

autoridades e da população, aceitação e estímulo do uso de drogas lícitas, falta de programas preventivos, desinformação, desemprego, desestruturação familiar).

Compreensão da família do usuário de drogas: $86,3 \%$ relatam que a família é desestruturada (conflitos conjugais; pais rigorosos, permissivos, ausentes, alcoolistas; mães protetoras, permissivas, ausentes, sedutoras; laços afetivos enfraquecidos; necessidade de um bode expiatório; estão doentes, codependentes, querem a internação do filho, rejeição parental); 13,6\% assinalam que a família não tem responsabilidade pelo uso de drogas do filho (o comprometimento psicológico familiar é pouco superior à média; não tem culpa, a desestruturação vem do uso de drogas; é um erro culpar a família).

Compreensão da postura da sociedade brasileira em relação às drogas e aos usuários: 72,2\% hipócrita, preconceituosa, discriminadora, acusadora; 36,6\% mal-informada, desinteressada, pânico e interesse diante de casos divulgados pela mídia; 4,5\% há crescente atenção na prevenção.

Mudanças de compreensão da farmacodependência no transcurso da experiência: $13,6 \%$ não houve mudanças; $22,7 \%$ substituição de preconceito pelo conceito de doença; $18,2 \%$ maior aceitação e respeito pelo sofrimento; $4,5 \%$ antes eu não sabia o que era dependência química; 4,5\% a abstinência não é cura; $9 \%$ fazer uma avaliação mais criteriosa; $4,5 \%$ deixar de ser um anteparo em relação à droga; 4,5\% trabalhar a compulsão é muito difícil; 4,5\% discriminar entre uso, abuso e dependência; $4,5 \%$ avaliar se há indicação de análise (tratamento psicanalítico).

\section{Discussão}

As dificuldades decorrentes da rotina de trabalho, aliadas à falta do hábito de integrar assistência e pesquisa em nossa cultura de serviços públicos e privados, fora do âmbito acadêmico, podem explicar as limitações de evolução teórico-técnica no tratamento de transtornos relacionados a substâncias entre os profissionais de nossa amostra. A negligência, verificada nas atividades de pesquisa, pode ser atribuída a falhas de formação acadêmica e ao desinteresse institucional em apoiar o trabalho de supervisão, estudos clínicos e de investigação sistemática da população atendida.

Há uma extensa literatura científica sobre os transtornos relacionados a substâncias, tais como a Revista Britânica de Adições, com mais de cem anos, revistas especializadas, em vários países, publicadas por órgãos científicos de reconhecido valor. A maioria dos profissionais entrevistados não se mostrou voltada para essas leituras. 


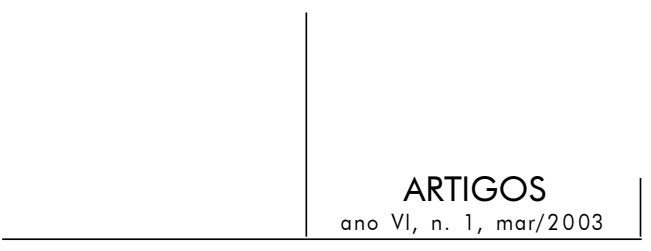

No presente estudo, verificamos que, se há profissionais que focalizam a intervenção clínica no combate ao uso de drogas (modelos de AA e NA, nos quais se acredita que a compulsão determina a falta de controle sobre o uso da substância e prega-se a abstinência), há outros, que procuram centralizar a abordagem terapêutica na totalidade dos aspectos da personalidade. Se alguns enfrentam a angústia decorrente do atendimento ao toxicodependente, condenando a droga, às vezes de forma ingênua e simplista, outros profissionais se esquivam deste estado psicológico. Entretanto, a angústia de atender pessoas com hábitos de risco permanece, e podemos observá-la na maioria dos entrevistados que trabalham há mais tempo com essa população. Os profissionais iniciantes, com meses ou alguns anos de experiência, ou os que não se consideram especialistas ou referências, encontram-se menos frustrados e com menor carga de ressentimento para com seus pacientes. Consideramos que esses profissionais, embora não tenham uma formação diferenciada para tratar transtornos relacionados a substâncias, demonstram uma atitude mais isenta para abordálos; o que pode favorecer o estabelecimento da relação terapêutica. Por outro lado, os chamados especialistas nem sempre conseguem manter-se livres de expectativas e com bem-estar psicológico, indispensáveis ao desenvolvimento da relação terapêutica. Verificamos, também, que os profissionais de enfermagem ou aqueles que fazem atendimentos mais breves, e freqüentemente com menor envolvimento pessoal, estão menos insatisfeitos com seus resultados.

É muito comum os profissionais de saúde mental revelarem resistência ou mesmo indisponibilidade para prestarem atendimento a pessoas que apresentam transtornos relacionados com uso de substâncias psicoativas. $\mathrm{O}$ argumento mais comum é de que se trata de pacientes que frustram, ou de que são "sociopatas". Assim é que, em cada uma das unidades de saúde mental (de São José dos Campos), há um profissional que é reconhecido como aquele que atende drogados e que tem experiência com essa população. Muitas vezes este profissional foi escalado pelos demais para ocupar tal lugar, e desobrigando-os, destarte, de realizar atendimentos perigosos e (ou) frustrantes. Os escalados para o que podemos chamar de missão ou se sentem confortáveis com o status institucional ou se tornam "prisioneiros" dos colegas.

Há indícios de que o propalado risco de se trabalhar com drogados é similar ao medo de se entrar em contato com pessoas estigmatizadas como loucas. Historicamente, o doente mental foi, e continua sendo objeto, de fantasias, discriminações, e exclusão social. Da mesma forma, o usuário de drogas, na atualidade, também passou a ser um dos representantes das possessões demoníacas medievais. Sabe-se que sua exclusão ocorre em várias instituições: família, escola, trabalho. Uma parte dos entrevistados fornecem indícios de que alguns profissionais de saúde são parte atuante no processo de afastamento desses 


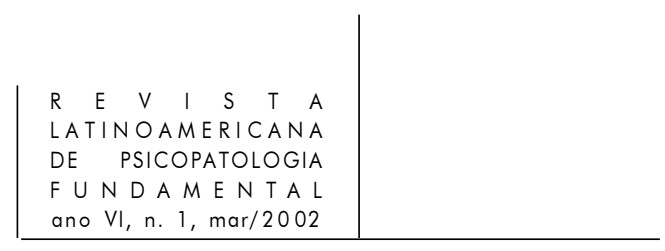

pacientes. Não podemos negligenciar a existência de riscos no atendimento a toxicômanos, entretanto, o mesmo cuidado deve estar presente nas demais situações vivenciadas nos serviços de saúde mental. Admitimos que a hipótese de resistências dos profissionais ao atendimento dessa população, foi confirmada pelos sujeitos de nossa pesquisa.

As explicações simplistas, de caráter biológico, psicológico ou psicanalítico, sobre a toxicomania, podem ser reflexo do postulado de que a doença mental está totalmente encerrada e localizada dentro do sujeito que a manifesta. $\mathrm{Na}$ literatura científica, há diferentes explicações para a etiologia da drogadependência. São elas: predisposição genética, particularidades bioquímicas, fragilidades egóicas, rejeição parental, desestruturação familiar, falhas de caráter ou de personalidade com tendências destrutivas, aumento da oferta e das facilidades para encontrar produtos psicoativos, desenvolvimento de produtos sintéticos com crescente poder de provocar dependência psicológica e (ou) psíquica, crise de valores e de referências éticas e falta de perspectivas de bem-estar social.

Estas explicações, mais habituais, e suas deformações interpretativas tiveram influência na criação de uma pseudocultura científica, dominada por crenças e dogmatismo, o que dificulta a apreensão mais ampla das dimensões biológica, psicológica e sociocultural que permeiam o fenômeno da adição química. Os profissionais que tratam de dependentes ficam de tal modo envolvidos em sua prática cotidiana que podem deixar de observar as várias formas de relação que as pessoas estabelecem com as drogas, desenvolvendo, assim, generalizações indevidas. Sabe-se que os pacientes, dos hospitais psiquiátricos e das unidades de saúde mental, com diagnóstico de toxicomania representam uma parcela pequena do universo populacional de utilizadores de drogas. Desta forma, os profissionais passam a enxergar o mundo social e humano a partir da perspectiva dos casos clínicos que atendem.

Muitas das abordagens dos profissionais pesquisados foram equivalentes às do senso comum, isto é, críticas, generalistas, sem discriminação quanto ao tipo de droga consumida - maconha, cocaína, "crack", álcool, etc - nem quanto à forma de uso. Todas recebem a mesma inscrição - "drogas" - com destino garantido, a dependência. O contexto em que ocorre a utilização do produto, a fase de desenvolvimento do usuário (infância, adolescência), avaliação de suas relações familiares, pessoais, profissionais e (ou) escolares, estudo de personalidade e dados biológicos são menos relevantes do que o conceito-drogas. Esta extrapolação de sua condição de sinal e de sintoma individual e social para doença psiquiátrica ou de desvio de comportamento, com vida própria e independente de seu processo gerador, ainda é o pensamento hegemônico. Tal pensamento predomina, embora pesquisas na área de abuso de substâncias, das últimas décadas, não tenham encontrado perfis específicos de personalidade ou 


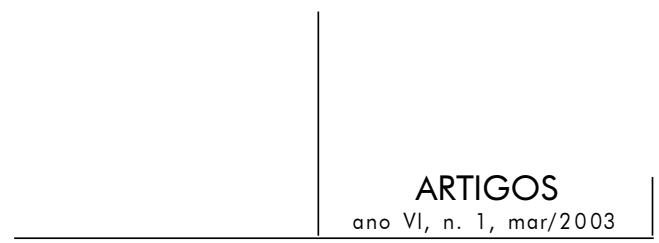

de famílias de dependentes de drogas. Além disso, sabe-se que o fenômeno também não se restringe a classes sociais, etnias, ou economias específicas (Rezende, 1999).

\section{Conclusões}

As dificuldades encontradas pelos profissionais do serviço público são de várias ordens: excesso de trabalho, falta de supervisão como rotina inerente ao trabalho em saúde mental, falhas de formação ou mesmo total falta para efetuar as intervenções técnicas propostas, tais como psicoterapia de grupo ou, ainda, para compreender as complexas questões sociais, psicológicas e médicas que envolvem os transtornos relacionados às substâncias psicoativas. O empenho dos profissionais, da mesma forma que as suas angústias, fica percebido durante as conversas. Os diálogos com os entrevistados indicaram que há insatisfação com os resultados obtidos e com as condições de trabalho. Praticamente não há cursos de reciclagem, controle de eficácia, ou follow-up. A improvisação e o uso de técnicas ecléticas predominam sobre as intervenções definidas e avaliadas sistematicamente. A experiência assistencial é dominante em relação a de pesquisa, ou de atualização da literatura científica especializada.

\section{Referências}

Bardin, L. Análise de conteúdo. Trad. Luís Antero Reto e Augusto Pinheiro. Lisboa: Edições 70, 1977.

Bergeret, J. Manual de psicologia patológica. Trad. Washington Loyello. São Paulo: Masson, 1983.

ReZEnde, M.M. Tratamento de dependentes de drogas: diálogos com profissionais da área de saúde mental. Tese (Doutorado). Faculdade de Ciências Médicas, Universidade Estadual de Campinas, 1999.

\section{Resumos}

Esta investigación fue realizada con profesionales de salud mental de diversas formaciones de nivel universitario que atienden dependientes de drogas en las ciudades de Taubaté y São José dos Campos, situadas en el valle de Paraíba, en el estado de San 
Pablo. Se investigaron las características relativas a la formación; a la actuación y a algunos de los puntos de vista y observaciones de estos profesionales sobre la experiencia de atención a pacientes que presentan trastornos relacionados a substancias. Los diálogos con los entrevistados indicaron que hay insatisfacción con los resultados obtenidas y con las condiciones de trabajo. Prácticamente no hay cursos de reciclaje, control de eficacia o follow-up. La improvisación y el uso de técnicas eclécticas predominan por sobre las intervenciones definidas y evaluadas sistemáticamente. La experiencia asistencial es dominante en comparación con la investigación o a la actualización de la literatura científica especializada.

Palabras clave: profesionales de salud mental, experiencia, atención de pacientes, dependencia de drogas

Cette recherche a été réalisée avec des professionnels de Santé Mentale de formations universitaires diverses qui s'occupent de patients toxicomanes dans les villes de Taubaté et São José dos Campos, localisées dans le Vale de Paraib, Etat de São Paulo. Nous avons procédé à l'investiguation des caractéristiques relative à la formation, à l'activité ,certains des points de vue et observations de ces professionnels à propos de l'expérience de prise en charge de patients présentants des troubles relatifs à la prise de substances chimiques. Le dialogue avec les personnes interrogées indiquent un insatisfaction notable en ce qui concerne les résultats obtenues et les conditions de travail. Il n'y a pratiquement pas de formation permanente, ni contrôle, ni de suivi. L'improvisation et l'utilisation de techniques éclectiques prédominent par rapport aux interventions définies et évaluées systématiquement. L'expérience d'assistance prédomine sur la recherche, ou l'actualisation de la lecture scientifique spécialisée.

Mots clés: Professionnels de la Santé Mentale, expérience, prise en charge, toxicomanes

The research described in this article was carried out on mental health professionals, holders of degrees in different areas, who deal with drug dependents in the cities of Taubaté and São José dos Campos, State of São Paulo, Brazil. Characteristics related to educational background, performance, opinions and observations of these professionals are studied with regard to the assistance provided to drug-addicted patients. The dialogues with the interviewees show that there is dissatisfaction with both the results obtained and the working conditions. There is virtually no on-going training, control of efficacy, or follow up. Improvisation and the use of eclectic techniques predominate over systematically defined and assessed interventions. The experience in assisting patients prevails over research and updating through specialised bibliography.

Key words: Mental Health, professionals, experience, assistance, drug-addicts

Versão inicial recebida em setembro de 2002

Versão revisada recebida em fevereiro de 2003 